\title{
Antibody-Drug Conjugates Used in Breast Cancers
}

\author{
Aram J. Abbas $\mathbb{D}^{1},{ }^{1}$ Marah F. Ibrahim $\mathbb{D}^{2}$, and Maher S. Saifo $\mathbb{D}^{1,2}$ \\ ${ }^{1}$ Faculty of Medicine, Damascus University, Damascus, Syria \\ ${ }^{2}$ Faculty of Pharmacy, Alsham Private University, Damascus, Syria \\ Correspondence should be addressed to Aram J. Abbas; aram.abbas.sy@gmail.com and Maher S. Saifo; maher.saifo@aspu.edu.sy
}

Received 13 March 2021; Revised 30 April 2021; Accepted 7 June 2021; Published 28 June 2021

Academic Editor: Nicola Silvestris

Copyright ( $\odot 2021$ Aram J. Abbas et al. This is an open access article distributed under the Creative Commons Attribution License, which permits unrestricted use, distribution, and reproduction in any medium, provided the original work is properly cited.

The prognosis of breast cancer has radically changed in recent years and continues to improve due to the broad application of effective therapies. New targeting strategies including targeted delivery of cytotoxic drugs via receptor-targeting agents have been developed. We summarize recent publications and developments of novel antibody-drug conjugates (ADCs) used to control breast cancer.

\section{Introduction}

Cancer is the second main cause of mortality worldwide [1]. Breast cancer is the most common cancer in women, and the most common cancer overall [2]. A subtype of breast cancer overexpresses HER2 receptors and is called HER2-positive (HER2+); HER 2+ breast cancer accounts for $15-20 \%$ of all breast cancers and is associated with poor patient outcome and aggressive phenotype [3]. For many years, the therapies of the breast cancer were based on known biomarkers such as estrogen receptor (ER), progesterone receptor (PR), and human epidermal growth factor receptor 2 (HER2) [4-6]. One of these treatments is trastuzumab; a humanized monoclonal antibody that targets HER2 receptor, leading to angiogenesis inhibition, diminished microvessel density, and better overall survival rates in patients with HER2positive breast cancer [7]. However, resistance to trastuzumab has been reported $[8,9]$, in addition to several severe adverse effects such as cardiac toxicity [10]. In general, naked monoclonal antibodies, despite their importance in cancer research, have not offered the expected curative results, so the need for more potent agents was clear in order to completely treat cancer. Further studies using monoclonal antibodies as a treatment were made [11] introducing us to the amazing therapeutic properties of them, especially the selective delivery of cytotoxic agents to tumor cells, creating what is called antibody-drug conjugates (ADCs) as a solution to increase the therapeutic index of a cytotoxic chemical agent $[11,12]$. Although these HER2-targeting therapies have improved the overall survival rate, many more cases are still not affected by these treatments. A large population of them have breast cancer which does not overexpress HER2 receptors, those are clinically categorized as "HER2-negative." The word "negative" does not mean that the tumor does not express any HER2 receptors; it actually means that the amount of the receptors is not enough for the anti-HER2 antibodies to be used as a treatment [13]. If the tumor also does not express hormone receptors (HR), then it is called triple negative breast cancer (TNBC). In this review, we are going to describe ADCs generally and ADCs used in managing breast cancers specifically.

\section{Antibody-Drug Conjugates (ADCs)}

ADCs are a new class of protein-based therapeutic agents which bring together the targeting capabilities, high selectivity, and stability of $\mathrm{mAbs}$ with the cancer-killing potential of highly potent payloads to increase precise drug delivery in cancer cells, while sparing healthy tissues and/or cells from chemotherapeutic damage. This ability of discrimination between normal and cancerous cells would not have been achieved without decades of development of mAbs [9, 14-18]. 


\section{Antibody-Drug Conjugates (ADCs) Structure}

In order to achieve the desired results, each ADC must contain three parts (Figure 1) [19]:

Monoclonal antibody: it binds the ADC to a specific tumor cell surface protein [19]. The antibody should bind tumor cells with high avidity and have little crossreactivity with healthy cells so that it does not affect them. All the antibodies developed or currently in clinical trials are immunoglobulin G ( $\operatorname{IgG}$ ); taking advantage of that, they contain multiple native sites for conjugation and can be modified for additional reactive sites [20, 21]. Most of the ADCs are built on IgG1 scaffolds because the antibodydependent cell mediated cytotoxicity (ADCC) and complement-dependent cytotoxicity (CDC) are much stronger in IgG1 and IgG3 than IgG2 and IgG4 [22, 23].

Linker: it is a chemical spacer between the cytotoxic drug and the monoclonal body. It is usually stable in the circulation, but most of the linkers are easily displaced inside the cells. However, if the linker stays stable inside the cell, it requires degradation to release the drug. The linker must allow the release of the drug in its active form within or close enough to the target cells, because early release of drugs in the circulation can result in systemic toxicity and a lower therapeutic index $[24,25]$. There are 2 types of linkers: cleavable and noncleavable, both of them are used in the ADCs developed or currently in clinical trials [26]. Cleavable linkers depend on the differences between conditions in the bloodstream and the cytoplasmic conditions within cancer cells (low $\mathrm{pH}$, proteolytic cleavage, and high glutathione concentrations). Depending on their response to intracellular conditions, there are three types of cleavable linkers: hydrazone, disulfide, and peptide linkers $[16,27]$. On the other hand, noncleavable linkers release the drug after internalization in the target cell $[16,27]$; they rely on complete proteolytic degradation of the antibody to the amino acid level within the lysosome [27], that means they require appropriate internalization and degradation inside the cell to be active. The most common example of noncleavable linkers is the thioether linker.

Cytotoxic drug: cytotoxic compounds are divided into two main categories: microtubule inhibitors and DNAdamaging agents. There are also other small molecules under investigation [27, 28]. All the cytotoxic compounds used in the ADC structure must have higher toxic potency compared with standard chemotherapeutic agents, be able to kill cancer cells by induction of apoptosis, have a suitable functional group for linkage to an antibody, and be soluble in aqueous solutions to enable the reaction with antibodies $[24,29,30]$.

\section{Development of Antibody-Drug Conjugates}

There are three generations of ADCs: first, second, and third. Table 1shows the main differences between them.

\section{Mechanism of Action of HER2-Directed ADCs [34]}

5.1. Classical Mode of Action. The monoclonal anti-HER2 binds to the HER2 expressed on the cells of the tumor and gets internalized by endocytosis. The proteases in the lysosomes cleavage the linker, releasing the payload and starting the cytotoxic effects.

5.2. Bystander Killing Effect. This effect happens when the ADC is designed that the antibody releases the payload before internalization so that the surrounding cells get affected by its cytotoxic effects even if they do not express the receptor.

\section{Antibody-Drug Conjugates (ADCs) Targeting HER2+ Receptors}

6.1. A166 [35]

(1) Monoclonal antibody: a human epidermal growth factor receptor 2 (EGFR2; HER2; ERBB2) targeting monoclonal antibody.

(2) Payload: an undisclosed cytotoxic agent with potential antineoplastic activity.

(3) Development status: first in human phase I/II.

6.2. ADCT-502 [36]

(1) Monoclonal antibody: an engineered version of the humanized monoclonal antihuman epidermal growth factor receptor 2 (HER2) immunoglobulin G1 (IgG1) trastuzumab.

(2) Linker: cathepsin B-cleavable valine-alanine linker.

(3) Payload: DNA cross-linking pyrrolobenzodiazepine (PBD) dimer-based drug tesirine.

(4) Development status [37]: phase I.

6.3. ALT-P7 [38]

(1) Monoclonal antibody: trastuzumab biobetter HM2.

(2) Payload: monomethyl auristatin E (MMAE).

(3) Development status [34]: phase I.

\subsection{Anti-HER2-vc0101 [39]}

(1) Monoclonal antibody: a human epidermal growth factor receptor 2 (HER2) site-specifically targeting monoclonal antibody.

(2) Linker: cleavable valine-citrulline- linker.

(3) Payload: an analog of dolastatin 10, auristatin-0101.

\subsection{ARX788 [40]}

(1) Monoclonal antibody: a human epidermal growth factor receptor 2 (EGFR2; HER2) site-specifically targeting monoclonal antibody.

(2) Linker: para-acetyl-phenylalanine ( $\mathrm{pAcF}$ linked to a nonnatural amino acid linker.

(3) Payload: auristatin analog and potent microtubule inhibitor monomethyl auristatin F (MMAF).

(4) Development status [41]: preclinical studies, phase I. 


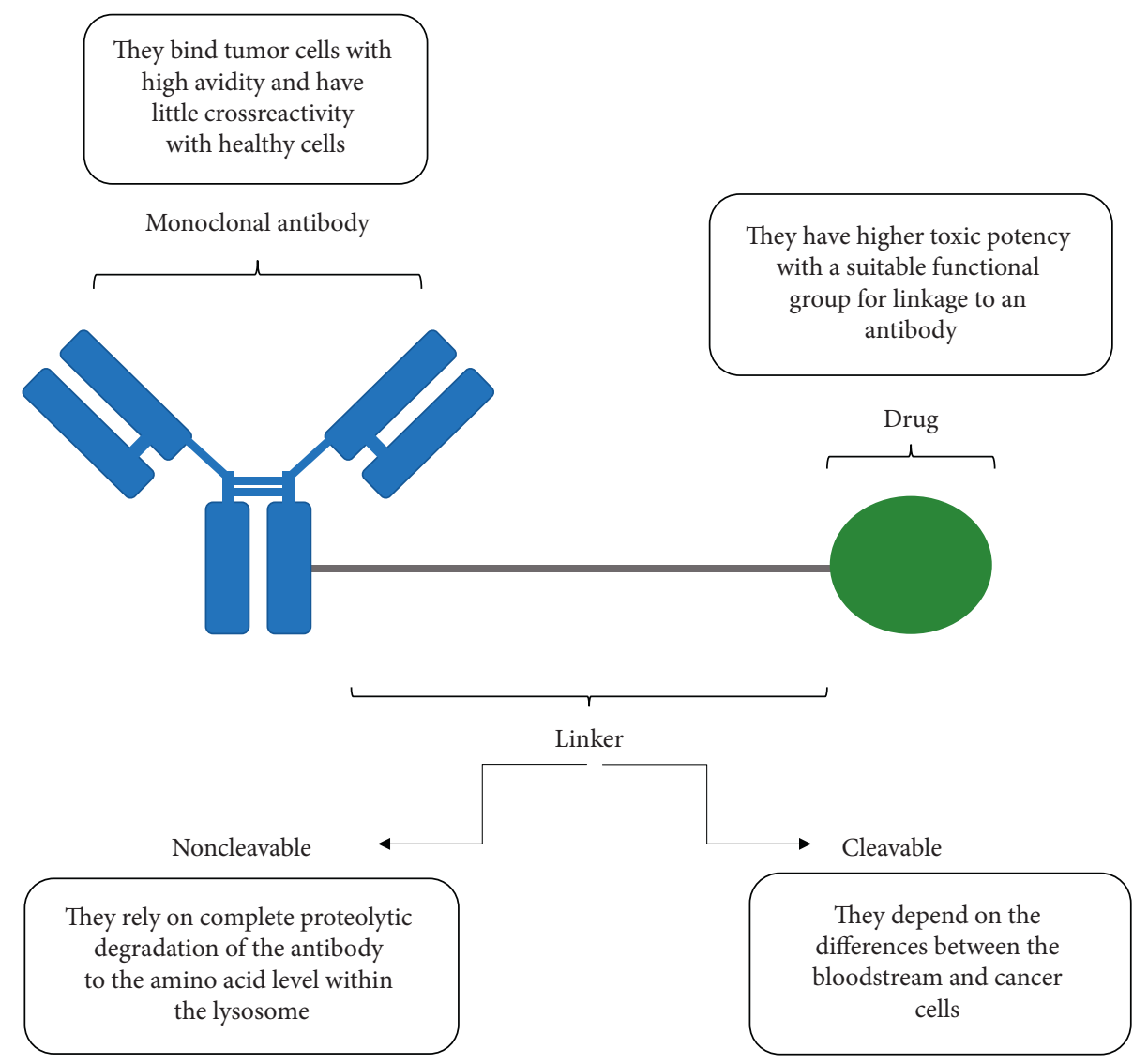

Figure 1: Structure of an antibody-drug conjugate [19].

TABLE 1: Main characteristics of the ADC generations.

\begin{tabular}{|c|c|c|}
\hline First-generation $[29,31]$ & Second-generation $[29,32]$ & Third-generation [33] \\
\hline $\begin{array}{l}\text { (1) Anticancer drugs were coupled } \\
\text { through noncleavable linkers to murine } \\
\text { mAbs. } \\
\text { (2) Evaluations showed that they were } \\
\text { only moderately potent and less active } \\
\text { than the parent drugs. } \\
\text { (3) Examples: KS1/4-methotrexate and } \\
\text { BR96-doxorubicin. }\end{array}$ & $\begin{array}{l}\text { (1) Huge improvements in mAbs technology were } \\
\text { made, increasing selective binding to tumor cells } \\
\text { and reducing crossreactivity with healthy cells. } \\
\text { Payloads with smaller molecules were also } \\
\text { discovered. } \\
\text { (2) Examples: brentuximab vedotin, ado- } \\
\text { trastuzumab emtansine, and inotuzumab } \\
\text { ozogamicin. }\end{array}$ & $\begin{array}{l}\text { (1) Site-specific conjugation was } \\
\text { developed, improving the therapeutic } \\
\text { index, stability, and potency. } \\
\text { (2) Examples: MEDI4276, vadastuximab } \\
\text { talirine, and IMGN779. }\end{array}$ \\
\hline
\end{tabular}

\subsection{BAT8001 [42]}

(1) Monoclonal antibody: a human epidermal growth factor receptor 2 (EGFR2; HER2; ErbB2) targeting monoclonal antibody.

(2) Payload: undisclosed maytansine derivative.

(3) Development status [43]: BAT8001 is in phase III clinical evaluation as a treatment of HER2-positivemetastatic breast cancer that is treated previously with trastuzumab.

\subsection{DHES0815A [44]}

(1) Monoclonal antibody: a monoclonal antibody targeting human epidermal growth factor receptor 2 (ERBB2; EGFR2; HER2).
(2) Payload: a DNA minor groove cross-linking agent pyrrolo[2, 1-c][1, 4]benzodiazepine monoamide (PBD-MA).

(3) Development status [34]: first-in-human (FIH), phase I, open-label, multicenter, dose-escalation study.

\subsection{Disitamab Vedotin [45]}

(1) Monoclonal antibody: a monoclonal antibody targeting human epidermal growth factor receptor 2 (ERBB2; EGFR2; HER2).

(2) Linker: a cleavable maleimidocaproyl-valyl-citrullinyl-p-aminobenzyloxycarbonyl (mc-val-citPABC) type linker.

(3) Payload: monomethyl auristatin E (MMAE). 
(4) Development status: phase I and phase II .

\section{9. $L C B 14-0110$ [46]}

(1) Monoclonal antibody: a monoclonal antibody against human epidermal growth factor receptor 2 (HER2) site-specifically.

(2) Payload: monomethyl auristatin F (MMAF).

(3) Development status: LegoChemistry ${ }^{\mathrm{TM}}$ and ADC platform technology ConjuAll ${ }^{\mathrm{TM}}$.

\subsection{Hertuzumab Vedotin [47]}

(1) Monoclonal antibody: hertuzumab.

(2) Payload: monomethyl auristatin E (MMAE).

(3) Development status: phase I and phase II.

\subsection{MEDI4276 [48]}

(1) Monoclonal antibody: a bispecific antibody against the extracellular domain of human epidermal growth factor receptor 2 (HER2; ERBB2) comprised of the single-chain variable fragment $(\mathrm{scFv})$ of the antiHER2 monoclonal antibody trastuzumab, fused to the heavy chains of the anti-HER2 monoclonal antibody 39S.

(2) Payload: tubulysin

(3) Development status [49]: phase I.

\subsection{MI130004 [50]}

(1) Monoclonal antibody: trastuzumab.

(2) Linker [51]: it has a maleimide group to facilitate conjugation to Cys residues.

(3) Payload: PM050489.

(4) Development status [51]: preclinical.

6.13. $M M-302$ [52]

(1) Monoclonal antibody: a monoclonal antibody against the human epidermal growth factor receptor 2 (HER2).

(2) Payload: the antineoplastic anthracycline antibiotic doxorubicin encapsulated within liposomes.

(3) Development status [53]: phase II.

\subsection{Trastuzumab Deruxtecan [54]}

(1) Monoclonal antibody: a monoclonal antibody targeting human epidermal growth factor receptor 2 (ERBB2; EGFR2; HER2).

(2) Linker: a tetrapeptide linker, Gly-Phe-Leu-Gly (GFLG).

(3) Payload: deruxtecan, a derivative of the camptothecin analog exatecan (DXd; DX-8951 derivative); a
DNA topoisomerase 1 (topoisomerase I; Top1) inhibitor, with antineoplastic activity.

(4) Development status [55]: FDA approval based on the results of the registrational phase II trial DESTINYBreast01.

\subsection{Trastuzumab Duocarmazine [56]}

(1) Monoclonal antibody: trastuzumab, a monoclonal antibody targeting epidermal growth factor receptor 2 (HER2).

(2) Linker [57]: a cleavable linker N-[2-(2maleimidoethoxy)ethoxycarbonyl]-L-valyl-L-citrullinyl-p-aminobenzyloxycarbonyl-N-[2-(2hydroxyethoxy)ethyl]-N-[2-(methylamino)ethyl] carbamoyl.

(3) Payload: the duocarmycin prodrug.

(4) Development status [57]: phase I, phase II, and phase III.

6.16. Trastuzumab Emtansine [58]

(1) Monoclonal antibody: trastuzumab.

(2) Linker [59]: noncleavable succinimidyl-4-(N-maleimidomethyl) cyclohexane-1-carboxylate (SMCC) linker

(3) Payload: the microtubule-inhibitory agent DM1.

(4) Development status [59]: approved 2013 and approved 2019.

6.17. XMT-1522 [60]

(1) Monoclonal antibody: HT-19, a monoclonal antibody directed against the human epidermal growth factor receptor 2 (ERBB2; HER2) that binds to domain IV of HER2 to an epitope that is distinct from the trastuzumab-binding site

(2) Payload: proprietary auristatin-derived payload molecules.

(3) Development status [61]: in January 2019, phase I studies for breast cancer (late-stage disease, metastatic disease), gastric cancer (late-stage disease), and nonsmall cell lung cancer (late-stage disease) are discontinued (United States)

\section{Antibody-Drug Conjugates (ADCs) Targeting Triple Negative Breast Cancer (TNBC)}

\subsection{Sacituzumab Govitecan [62]}

(1) Monoclonal antibody: sactizumab, an anti-Trop-2 humanized antibody.

(2) Payload: the topoisomerase-I inhibitor SN-38.

(3) Development status [63]: phase I/II. 


\subsection{Ladiratuzumab Vedotin [64]}

(1) Monoclonal antibody: the zinc transporter LIV-1 targeting humanized antibody.

(2) Payload: monomethyl auristatin E (MMAE).

(3) Development status: phase I.

\subsection{AVID100 [65]}

(1) Monoclonal antibody: anti-EGFR antibody.

(2) Payload: DM1 (derivative of maytansine).

(3) Development status: phase I.

\subsection{U3-1402 [66]}

(1) Monoclonal antibody: an anti-HER3 antibody.

(2) Payload: a topoisomerase I inhibitor exatecan derivative (DXd).

(3) Development Status: phase I/II.

\subsection{CAB-ROR2-ADC [67]}

(1) Monoclonal antibody: receptor tyrosine kinase-like orphan receptor 2 (ROR2) targeting antibody.

(2) Payload: an undisclosed payload.

(3) Development status: phase I/II.

\subsection{Anti-CA6-DM4 Immunoconjugate [68]}

(1) Monoclonal antibody: a humanized DS6 antibody directed against tumor-associated sialoglycotope CA6.

(2) Payload: the maytansinoid DM4.

(3) Development status: phase I.

\section{Conclusion}

Breast cancer has become the most common cancer in the world; a lot of treatment methods and technologies were used in order to control it, but all of them did not achieve the required goal, until the invention of the antibody-drug conjugates. The concept of targeted delivery of anticancer drugs helped the oncologists to improve the tumor selectivity of anticancer drugs and to lower their systemic toxicity. Meaning that these drugs could be administered at higher doses, providing better therapeutic benefit to their patients. The tumor selectivity of antibodies offered a chance to achieve this goal by using them as guide for the drug towards the tumor. This seemingly simple concept had great attention from researchers at academic institutions and in the pharmaceutical industry. The current breed of ADCs uses antibodies that are humanized, not immunogenic, and linkers that are designed to be stable in circulation, but are cleaved upon delivery into a cell. The recent FDA approvals of new ADCs have generated tremendous excitement. There are a lot of ADCs currently in clinical evaluation and almost every major pharmaceutical company has embraced this technology. There is active research by medicinal chemists to develop new linkers and discover new potent effector molecules suitable for use in ADCs, while biologists have focused on identifying cell-surface targets suitable for antibody development.

\section{Conflicts of Interest}

The authors declare that there are no conflicts of interest regarding the publication of this paper.

\section{References}

[1] World Health Organization, Cancer, World Health Organization, Berlin, Germany, 2018, https://www.who.int/newsroom/fact-sheets/detail/cancer.

[2] H. Sung, "Global cancer statistics 2020: GLOBOCAN estimates of incidence and mortality worldwide for 36 cancers in 185 countries," CA: A Cancer Journal for Clinicians, vol. 71, no. 1, 2021.

[3] D. Slamon, G. Clark, S. Wong, W. Levin, A. Ullrich, and W. McGuire, "Human breast cancer: correlation of relapse and survival with amplification of the HER-2/neu oncogene," Science, vol. 235, no. 4785, pp. 177-182, 1987.

[4] R. L. Siegel, K. D. Miller, and A. Jemal, "Cancer statistics, 2020," CA: A Cancer Journal for Clinicians, vol. 70, no. 1, pp. 7-30, 2020.

[5] Y.-S. Sun, Z. Zhao, Z.-N. Yang et al., "Risk factors and preventions of breast cancer," International Journal of Biological Sciences, vol. 13, no. 11, pp. 1387-1397, 2017.

[6] Y. Naito and T. Urasaki, "Precision medicine in breast cancer," Chinese Clinical Oncology, vol. 7, no. 3, p. 29, 2018.

[7] A. C. Pinto, F. Ades, E. de Azambuja, and M. Piccart-Gebhart, "Trastuzumab for patients with HER2 positive breast cancer: delivery, duration and combination therapies," The Breast, vol. 22, no. Suppl 2, pp. S152-S155, 2013.

[8] M. Hubalek, C Brunner, K Matthä, and C Marth, "Resistance to HER2-targeted therapy: mechanisms of trastuzumab resistance and possible strategies to overcome unresponsiveness to treatment," Wiener Medizinische Wochenschrift (1946), vol. 160, no. 19-20, pp. 506-512, 2010.

[9] D. Jackson, J. Atkinson, C. I. Guevara et al., "In vitro and in vivo evaluation of cysteine and site specific conjugated herceptin antibody-drug conjugates," PLoS One, vol. 9, no. 1, Article ID e83865, 2014.

[10] R. K. Murthy, S Loi, A Okines et al., "Tucatinib, trastuzumab, and capecitabine for HER2-positive metastatic breast cancer," The New England Journal of Medicine, vol. 382, no. 7, pp. 597-609, 2020.

[11] G. Köhler and C. Milstein, "Continuous cultures of fused cells secreting antibody of predefined specificity," Nature, vol. 256, no. 5517 , pp. $495-497,1975$.

[12] J. M. Lambert and C. Q. Morris, "Antibody-drug conjugates (ADCs) for personalized treatment of solid tumors: a review," Advances in Therapy, vol. 34, no. 5, pp. 1015-1035, 2017.

[13] V. Oganesyan, L. Peng, J. S. Bee et al., "Structural insights into the mechanism of action of a biparatopic anti-HER2 antibody," Journal of Biological Chemistry, vol. 293, no. 22, pp. 8439-8448, 2018.

[14] A. Beck, T. Wurch, C. Bailly, and N. Corvaia, "Strategies and challenges for the next generation of therapeutic antibodies," Nature Reviews Immunology, vol. 10, no. 5, pp. 345-352, 2010. 
[15] C. R. Behrens, E. H. Ha, L. L. Chinn et al., "Antibody-drug conjugates (ADCs) derived from interchain cysteine crosslinking demonstrate improved homogeneity and other pharmacological properties over conventional heterogeneous ADCs," Molecular Pharmaceutics, vol. 12, no. 11, pp. 3986-3998, 2015.

[16] S. O. Doronina, B. E. Toki, M. Y. Torgov et al., "Development of potent monoclonal antibody auristatin conjugates for cancer therapy," Nature Biotechnology, vol. 21, no. 7, pp. 778-784, 2003.

[17] G. M. Dubowchik and M. A. Walker, "Receptor-mediated and enzyme-dependent targeting of cytotoxic anticancer drugs," Pharmacology \& Therapeutics, vol. 83, no. 2, pp. 67-123, 1999.

[18] E. Wagner-Rousset, "Antibody-drug conjugate model fast characterization by LC-MS following IdeS proteolytic digestion," MAbs, vol. 6, no. 1, pp. 273-285, 2014.

[19] A. Thomas, B. A. Teicher, and R. Hassan, "Antibody-drug conjugates for cancer therapy," The Lancet Oncology, vol. 17, no. 6, 2016.

[20] J. R. McCombs and S. C. Owen, "Antibody drug conjugates: design and selection of linker, payload and conjugation chemistry," The AAPS Journal, vol. 17, no. 2, pp. 339-351, 2015.

[21] H. L. Perez, P. M. Cardarelli, S. Deshpande et al., “Antibodydrug conjugates: current status and future directions," Drug Discovery Today, vol. 19, no. 7, pp. 869-881, 2014.

[22] R. Jefferis, "Antibody therapeutics:," Expert Opinion on Biological Therapy, vol. 7, no. 9, pp. 1401-1413, 2007.

[23] J. G. Salfeld, "Isotype selection in antibody engineering," Nature Biotechnology, vol. 25, no. 12, pp. 1369-1372, 2007.

[24] R. V. J. Chari, "Targeted cancer therapy: conferring specificity to cytotoxic drugs," Accounts of Chemical Research, vol. 41, no. 1, pp. 98-107, 2008.

[25] J. Feld, S. K. Barta, C. Schinke, I. Braunschweig, Y. Zhou, and A. K. Verma, "Linked-in: design and efficacy of antibody drug conjugates in oncology," Oncotarget, vol. 4, no. 3, pp. 397-412, 2013.

[26] R. V. J. Chari, M. L. Miller, and W. C. Widdison, "Antibodydrug conjugates: an emerging concept in cancer therapy," Angewandte Chemie International Edition, vol. 53, no. 15, pp. 3796-3827, 2014.

[27] R. Gébleux and G. Casi, “Antibody-drug conjugates: current status and future perspectives," Pharmacology \& Therapeutics, vol. 167, pp. 48-59, 2016.

[28] G. Moldenhauer, A. V. Salnikov, S. Lüttgau, I. Herr, J. Anderl, and H. Faulstich, "Therapeutic potential of amanitin-conjugated anti-epithelial cell adhesion molecule monoclonal antibody against pancreatic carcinoma," JNCI: Journal of the National Cancer Institute, vol. 104, no. 8, pp. 622-634, 2012.

[29] F. Dosio, P. Brusa, and L. Cattel, "Immunotoxins and anticancer drug conjugate assemblies: the role of the linkage between components," Toxins, vol. 3, no. 7, pp. 848-883, 2011.

[30] L. A. Smets, "Programmed cell death (apoptosis) and response to anti-cancer drugs," Anti-Cancer Drugs, vol. 5, no. 1, pp. 3-9, 1994.

[31] N. Endo, Y Takeda, K Kishida et al., "Target-selective cytotoxicity of methotrexate conjugated with monoclonal antiMM46 antibody," Cancer Immunology, Immunotherapy: CII, vol. 25, no. 1, pp. 1-6, 1987.

[32] H. Donaghy, "Effects of antibody, drug and linker on the preclinical and clinical toxicities of antibody-drug conjugates," MAbs, vol. 8, no. 4, pp. 659-671, 2016.

[33] A. Beck, L. Goetsch, C. Dumontet, and N. Corvaïa, "Strategies and challenges for the next generation of antibody-drug conjugates," Nature Reviews Drug Discovery, vol. 16, no. 5, pp. 315-337, 2017.

[34] G. Rinnerthaler, S. P. Gampenrieder, and R. Greil, "HER2 directed antibody-drug-conjugates beyond T-DM1 in breast cancer," International Journal of Molecular Sciences, vol. 20, no. 5, 2019.

[35] U.S. National Institutes of Health, N.C.I, anti-HER2 Antibody-Drug Conjugate A166, U.S. National Institutes of Health, Bethesda, MD, USA, 2020, https://www.cancer.gov/ publications/dictionaries/cancer-drug/def/anti-her2antibody-drug-conjugate-a166? redirect $=$ true.

[36] U.S. National Institutes of Health, N.C.I, Trastuzumab/ Tesirine Antibody-Drug Conjugate ADCT-502, U.S. National Institutes of Health, Bethesda, MD, USA, 2020, https://www. cancer.gov/publications/dictionaries/cancer-drug/def/ trastuzumab-tesirine-antibody-drug-conjugate-adct-502.

[37] R. L. B. Costa and B. J. Czerniecki, "Clinical development of immunotherapies for HER2+ breast cancer: a review of HER2-directed monoclonal antibodies and beyond," NPJ Breast Cancer, vol. 6, no. 1, p. 10, 2020.

[38] U.S. National Institutes of Health, N.C.I, HM2/MMAE Antibody-Drug Conjugate ALT-P7, U.S. National Institutes of Health, Bethesda, MD, USA, 2020, https://www.cancer.gov/ publications/dictionaries/cancer-drug/def/hm2-mmaeantibody-drug-conjugate-alt-p7.

[39] U.S. National Institutes of Health, N.C.I, anti-HER2-vc0101 ADC PF-06804103, U.S. National Institutes of Health, Bethesda, MD, USA, 2020, https://www.cancer.gov/ publications/dictionaries/cancer-drug/def/anti-her2-vc0101adc-pf-06804103.

[40] U.S. National Institutes of Health, N.C.I, anti-HER2 Antibody-Drug Conjugate ARX788, U.S. National Institutes of Health, Bethesda, MD, USA, 2020, https://www.cancer.gov/ publications/dictionaries/cancer-drug/def/anti-her2antibody-drug-conjugate-arx788.

[41] L. Skidmore, S. Sakamuri, N. A. Knudsen et al., "ARX788, a site-specific anti-HER2 antibody-drug conjugate, demonstrates potent and selective activity in HER2-low and T-DM1-resistant breast and gastric cancers," Molecular Cancer Therapeutics, vol. 19, no. 9, pp. 1833-1843, 2020.

[42] U.S. National Institutes of Health, N.C.I, anti-HER2 Antibody-Drug Conjugate BAT8001, U.S. National Institutes of Health, Bethesda, MD, USA, 2020, https://www.cancer.gov/ publications/dictionaries/cancer-drug/def/anti-her2antibody-drug-conjugate-bat 8001 .

[43] S. Loi, A. Giobbie-Hurder, A. Gombos et al., "Pembrolizumab plus trastuzumab in trastuzumab-resistant, advanced, HER2positive breast cancer (PANACEA): a single-arm, multicentre, phase 1b-2 trial," The Lancet Oncology, vol. 20, no. 3, pp. 371-382, 2019.

[44] U.S. National Institutes of Health, N.C.I, anti-HER2/PBD-MA Antibody-Drug Conjugate DHES0815A, U.S. National Institutes of Health, Bethesda, MD, USA, 2020, https://www. cancer.gov/publications/dictionaries/cancer-drug/def/antiher2-pbd-ma-antibody-drug-conjugate-dhes0815a.

[45] ADC Review, "J.o.A.-d.C. Disitamab Vedotin | RC-48 | RC 480-ADC | RC48," 2020, https://www.adcreview.com/ drugmap/disitamab-vedotin/.

[46] ADC Review, "J.o.A.-d.C. LCB14-0110 (Herceptin-LC-LBGMMAF)," 2020, https://www.adcreview.com/drugmap/lcb140110-herceptin-lc-lbg-mmaf/.

[47] ADC Review, "J.o.A.-d.C. Hertuzumab Vedotin," 2020, https://www.adcreview.com/drugmap/hertuzumab-vedotin/. 
[48] U.S. National Institutes of Health, N.C.I, anti-HER2 Antibody-Drug Conjugate MEDI4276, U.S. National Institutes of Health, Bethesda, MD, USA, 2020, https://www.cancer.gov/ publications/dictionaries/cancer-drug/def/anti-her2antibody-drug-conjugate-medi4276.

[49] J. Y. Li, S. R. Perry, V. Muniz-Medina et al., "A biparatopic HER2-targeting antibody-drug conjugate induces tumor regression in primary models refractory to or ineligible for HER2-targeted therapy," Cancer Cell, vol. 29, no. 1, pp. 117-129, 2016.

[50] P. Avilés, J. M. Domínguez, M. J. Guillén et al., "MI130004, a novel antibody-drug conjugate combining trastuzumab with a molecule of marine origin, shows outstanding in vivo activity against HER2-expressing tumors," Molecular Cancer Therapeutics, vol. 17, no. 4, pp. 786-794, 2018.

[51] Conjugates, A.R.J.o.A.-D, MI130004, 2020, https://www. adcreview.com/drugmap/mil30004/.

[52] U.S. National Institutes of Health, N.C.I, HER2-targeted Liposomal Doxorubicin Hydrochloride MM-302, U.S. National Institutes of Health, Bethesda, MD, USA, 2020, https://www. cancer.gov/publications/dictionaries/cancer-drug/def/her2targeted-liposomal-doxorubicin-hydrochloride-mm-302.

[53] Conjugates, A.R.J.o.A.-D. MM-302, 2020, https://www. adcreview.com/drugmap/mm-302/.

[54] U.S. National Institutes of Health, N.C.I, Fam-Trastuzumab Deruxtecan-Nxki, U.S. National Institutes of Health, Bethesda, MD, USA, 2020, https://www.cancer.gov/ publications/dictionaries/cancer-drug/def/trastuzumabderuxtecan.

[55] Conjugates, A.R.J.o.A.-D. Trastuzumab deruxtecan, 2020, https://www.adcreview.com/trastuzumab-deruxtecan-drugdescription/.

[56] Z. Xu, D. Guo, Z. Jiang et al., "Novel HER2-targeting antibody-drug conjugates of trastuzumab beyond T-DM1 in breast cancer: trastuzumab deruxtecan(DS-8201a) and (Vic-) Trastuzumab duocarmazine (SYD985)," European Journal of Medicinal Chemistry, vol. 183, p. 111682, 2019.

[57] Conjugates, A.R.J.o.A.-D. Trastuzumab duocarmazine | SYD985 | Trastuzumab vc-seco-DUBA, 2020, https://www. adcreview.com/drugmap/trastuzumab-duocarmazinesyd $985 /$.

[58] G. D. Lewis Phillips, G. Li, D. L. Dugger et al., "Targeting HER2-positive breast cancer with trastuzumab-DM1, an antibody-cytotoxic drug conjugate," Cancer Research, vol. 68, no. 22, pp. 9280-9290, 2008.

[59] Conjugates, A.R.J.o.A.-D. Trastuzumab Emtansine | T-DM1 | Kadcyla ${ }^{\circledR}, \quad 2020$, https://www.adcreview.com/drugmap/ trastuzumab-emtansine-t-dm1-kadcyla/.

[60] V. Le Joncour, A. Martins, M. Puhka et al., "A novel antiHER2 antibody-drug conjugate XMT-1522 for HER2-positive breast and gastric cancers resistant to trastuzumab emtansine," Molecular Cancer Therapeutics, vol. 18, no. 10, pp. 1721-1730, 2019.

[61] Conjugates, A.R.J.o.A.-D. XMT-1522 | TAK-522, 2020, https://www.adcreview.com/drugmap/xmt-1522/.

[62] W. Dong, J. Shi, T. Yuan et al., "Antibody-drug conjugates of 7-ethyl-10-hydroxycamptothecin: sacituzumab govitecan and labetuzumab govitecan," European Journal of Medicinal Chemistry, vol. 167, pp. 583-593, 2019.

[63] A. Bardia, I. A. Mayer, L. T. Vahdat et al., "Sacituzumab govitecan-hziy in refractory metastatic triple-negative breast cancer," New England Journal of Medicine, vol. 380, no. 8, pp. 741-751, 2019.
[64] D. Sussman, L. M. Smith, M. E. Anderson et al., "SGN-LIV1A: a novel antibody-drug conjugate targeting LIV-1 for the treatment of metastatic breast cancer," Molecular Cancer Therapeutics, vol. 13, no. 12, pp. 2991-3000, 2014.

[65] A. Nagayama, N Vidula, L Ellisen, and A Bardia, "Novel antibody-drug conjugates for triple negative breast cancer," Therapeutic Advances in Medical Oncology, vol. 12, 2020.

[66] Y. Hashimoto, K. Koyama, Y. Kamai et al., "A novel HER3targeting antibody-drug conjugate, U3-1402, exhibits potent therapeutic efficacy through the delivery of cytotoxic payload by efficient internalization," Clinical Cancer Research, vol. 25, no. 23, pp. 7151-7161, 2019.

[67] M. Bayerlová, K. Menck, F. Klemm et al., "Ror2 signaling and its relevance in breast cancer progression," Frontiers in Oncology, vol. 7, p. 135, 2017.

[68] M. Trombe, "Abstract 235: preclinical activity of an antibody drug conjugate targeting tumor specificmucl structural peptide-glycotope," Experimental and Molecular Therapeutics, vol. 79, no. 13 Supplement, p. 235, 2019. 